\title{
Molecular subtypes of serous borderline ovarian tumor show distinct expression patterns of benign tumor and malignant tumor-associated signatures
}

\author{
Edward WJ Curry ${ }^{1}$, Euan A Stronach ${ }^{1}$, Nona R Rama ${ }^{1}$, Yuepeng YP Wang ${ }^{1}$, Hani Gabra ${ }^{1}$ and \\ Mona A El-Bahrawy ${ }^{2,3}$ \\ ${ }^{1}$ Ovarian Cancer Action Research Centre, Department of Cancer and Surgery, Imperial College London, \\ Hammersmith Hospital, London, UK; ${ }^{2}$ Department of Histopathology, Centre for Pathology, \\ Imperial College London, Hammersmith Hospital, London, UK and ${ }^{3}$ Department of Pathology, Faculty of \\ Medicine, University of Alexandria, Alexandria, Egypt
}

\begin{abstract}
Borderline ovarian tumors show heterogeneity in clinical behavior. Most have excellent prognosis, although a small percentage show recurrence or progressive disease, usually to low-grade serous carcinoma. The aim of this study was to understand the molecular relationship between these entities and identify potential markers of tumor progression and therapeutic targets. We studied gene expression using Affymetrix HGU133plus2 GeneChip microarrays in 3 low-grade serous carcinomas, 13 serous borderline tumors and 8 serous cystadenomas. An independent data set of 18 serous borderline tumors and 3 low-grade serous carcinomas was used for validation. Unsupervised clustering revealed clear separation of benign and malignant tumors, whereas borderline tumors showed two distinct groups, one clustering with benign and the other with malignant tumors. The segregation into benign- and malignant-like borderline molecular subtypes was reproducible on applying the same analysis to an independent publicly available data set. We identified $\mathbf{5 0}$ genes that separate borderline tumors into their subgroups. Functional enrichment analysis of genes that separate borderline tumors to the two subgroups highlights a cell adhesion signature for the malignant-like subset, with Claudins particularly prominent. This is the first report of molecular subtypes of borderline tumors based on gene expression profiling. Our results provide the basis for identification of biomarkers for the malignant potential of borderline ovarian tumor and potential therapeutic targets for low-grade serous carcinoma.
\end{abstract}

Modern Pathology (2014) 27, 433-442; doi:10.1038/modpathol.2013.130; published online 16 August 2013

Keywords: benign; borderline; malignant; molecular subtypes; ovary; tumor

Borderline ovarian tumors are a heterogeneous group of tumors with variable biological behavior, ${ }^{1}$ where the majority present with disease confined to the ovary and have an excellent prognosis after surgical removal. Approximately $15 \%$ of patients may subsequently have recurrent disease or progress to invasive carcinoma. ${ }^{2,3}$

Identifying the subset of borderline ovarian tumors with the potential for aggressive behavior would be

Correspondence: Dr MA El-Bahrawy, MBBCh, PhD, FRCPath, Department of Histopathology, Centre for Pathology, Imperial College London, Hammersmith Hospital, DuCane Road, London W12 0NN, UK.

E-mail: m.elbahrawy@imperial.ac.uk

Received 13 March 2013; revised 6 May 2013; accepted 11 May 2013; published online 16 August 2013 helpful in providing personalized approaches to management, regarding the extent of surgery, need for follow-up and targeted therapeutic strategies in recurrent borderline ovarian tumors. To date, there are no robust clinical, histological or molecular markers that distinguish high-risk cases. The only prognostic factor of progression to invasive disease is the subtype of peritoneal implants. ${ }^{1,4}$ Survival of patients with non-invasive implants was $95.3 \%$, as compared with $66 \%$ for invasive implants $(P<0.0001)$. Although not an independent predictor of worse prognosis, the presence of micropapillary architecture in the primary ovarian tumor is a strong predictor of invasive implants. There is controversy regarding the prognostic significance of stromal microinvasion in borderline ovarian tumors. Although some authors report that microinvasion was associated with a $100 \%$ 
survival rate at 6.7 years, ${ }^{5}$ others report that stromal microinvasion in the primary tumor correlated with adverse outcome. ${ }^{6,7}$

Recent studies suggested that the morphological features of the primary tumor and the peritoneal 'implants' can reliably subclassify serous borderline ovarian tumors into benign and malignant types, where tumors with micropapillary architecture are designated 'micropapillary serous carcinomas,' and those lacking these features, 'atypical proliferative serous tumors.' 5

In the dualistic model, epithelial ovarian tumors are divided into two categories (designated as type I and type II), which correspond to two pathways of tumorigenesis. Low-grade serous carcinoma is the prototypic type I tumor and high-grade serous carcinoma is the prototypic type II tumor. ${ }^{8}$ Low-grade serous carcinomas show frequent KRAS mutations and usually have an indolent course. Moreover, the carcinomas exhibit a shared lineage with the corresponding benign cystic neoplasm, often through an intermediate (borderline tumor) step, supporting the morphologic continuum of tumor progression. ${ }^{9}$ High-grade serous carcinomas are rarely associated with a precursor, and are aggressive and mostly display TP53 mutations but rarely KRAS mutations. ${ }^{8-12}$

Borderline ovarian tumors are understudied and poorly understood at the molecular level. ${ }^{13-15}$ Bonome et al $^{16}$ studied the genome-wide expression profile of borderline ovarian tumors and invasive ovarian cancers. Unsupervised hierarchical clustering analysis revealed a distinct separation between borderline ovarian tumors and high-grade lesions. The majority of low-grade tumors clustered with borderline ovarian tumors, suggesting that borderline ovarian tumors are distinct from high-grade carcinomas but remarkably similar to low-grade carcinomas. ${ }^{16}$ Furthermore, May et $a l^{17}$ have shown that borderline ovarian tumors of the micropapillary type have a gene expression profile more similar to low-grade serous carcinoma and distinct from borderline ovarian tumors without micropapillary features.

In this study, using the Affymetrix U133 Plus 2.0 oligonucleotide array, we investigated gene expression patterns in serous benign, borderline and low-grade malignant tumors. The identified differentially expressed genes revealed distinct molecular signatures of the benign and malignant subgroups and molecular subtypes of borderline ovarian tumors.

\section{Materials and methods}

\section{Tumor Samples}

Frozen tissue from 3 low-grade serous carcinomas (all stage III), 13 serous borderline ovarian tumors (Supplementary Table S1 summarizes the relevant histopathological features of the tumors) and 8 benign serous cystadenomas was obtained from the Human Biomedical Resource Centre at Hammersmith Hospital. Each sample contained at least $80 \%$ tumor tissue. Written informed consent for the use of human tissue samples in research was obtained from all patients. The study and use of human tissue was approved by Hammersmith and Queen Charlotte's and Chelsea Research Ethics Committee.

\section{Gene Expression Microarrays}

Gene expression levels were obtained for using Affymetrix HGU133plus2 GeneChip microarrays. Nucleic acid extraction, sample labeling, microarray hybridization and imaging were carried out by the Genome Institute of Singapore, following the manufacturer's instructions. Background correction, probelevel summarization and normalization of raw data from Affymetrix CEL files was performed across the full set of samples using Robust Multichip Average (RMA), ${ }^{18}$ as implemented in the 'Affy' package of Bioconductor.

An independent study ${ }^{19}$ was used to validate our observations. Raw data from Affymetrix HGU133plus2 GeneChip microarrays was obtained from the Gene Expression Omnibus with Accession number GSE9899 for borderline ovarian tumors and low-grade serous carcinoma.

\section{Cluster Analysis}

For unsupervised hierarchical clustering, probe sets with median log 2 expression values (following RMA normalization) $<5$ were filtered out. Any remaining probe sets with variances falling into the lowest $20 \%$ of probe-set variances across the data set were additionally filtered out. A correlation-based distance metric was calculated for each pair of samples and used as the basis of hierarchical clustering, defined as 1 minus the Pearson correlation coefficient between the vectors of expression values from each sample, for the probe sets passing the filter. Hierarchical clustering was performed using the 'hclust' function provided in $\mathrm{R}$, using complete linkage. The resulting sample clustering was confirmed by repeating the analysis using a Euclidean distance metric, which in all cases gave rise to the same structure as the clustering based on the correlation distance metric.

Gene expression heatmaps were produced in $\mathrm{R}$ using the 'heatmap.2' function provided in the 'gplots' package. Expression values in each row (ie for each gene) were scaled by subtracting the mean value for the corresponding gene across the samples included in the plot, and divided by the standard deviation of the values for the corresponding gene across the samples included in the plot. The columnwise dendrogram shown is the result of completelinkage hierarchical clustering based on the pairwise Euclidean distances of the scaled expression values. 


\section{Statistical Analysis}

Genes with malignancy-associated expression (ie genes more highly expressed in malignant tumors) were identified using LIMMA $^{20}$ to calculate empirical Bayes moderated t-statistics from the comparison of each gene's expression levels in the benign and malignant samples from the data set. The Benjamini-Hochberg approach to multiple testing adjustments was used to select a list of genes with a family-wise error rate of 0.05. Adjusted $P$-values from the moderated t-statistics were used to rank the genes in terms of the significance of their malignancy-associated differential expression across the data set.

To identify genes with significant differential expression between the subgroups of borderline ovarian tumors identified by hierarchical clustering, representative 'consensus' sets of samples for each subgroup were obtained by performing unsupervised hierarchical clustering on the full data set and the borderline ovarian tumors samples only, each with borderline ovarian tumor Euclidean distance and Pearson correlation as distance metrics. Consensus subgroups were defined as samples that clustered into the same group by all methods of clustering used, resulting in a set of five benign-like borderline ovarian tumors (ie borderline ovarian tumors clustering with benign serous cystadenomas; sample numbers 700 312, 700056,400 123, 600095 and 800084 ) and a set of five malignant-like borderline ovarian tumors (ie borderline ovarian tumors clustering with low-grade serous carcinomas; sample numbers $600381,400192,600308$, 600362 and 800148). There were three samples (800100, 600104 and 700135 ) that did not cluster so closely with either subgroup. In addition, two of these samples (600104 and 700135) clustered nearer the 'benign-like' subgroup over all genes, but with the 'malignant-like' subgroup when clustering on the basis of expression of the malignancyassociated genes. These were left out of the subgroup analyses to avoid confounding the results, justified principally by the fact that it would be difficult to interpret results if these samples were included. (See note in Discussion section for further comments on what these samples may represent.)

LIMMA was used to identify genes with significant differential expression between the two consensus subgroups. Significance was defined as Benjamini-Hochberg adjusted $P<0.05$ and at least twofold average expression difference between the subgroups.

For the Tothill et al data set, subgroups were identified following the same approach taken for our data set. This resulted in a set of five benign-like borderline ovarian tumors (Accession numbers GSM249714, GSM249716, GSM249717, GSM249726 and GSM249731) and a set of eight malignant-like borderline ovarian tumors (Accession numbers GSM249715, GSM249718, GSM249721, GSM249724,
GSM249725, GSM249728, GSM249729 and GSM249730). As observed with our data set, there were some borderline ovarian tumors (28\% compared with $23 \%$ in our own data set) that clustered into different subgroups when different clustering algorithms or distance metrics were used. These samples were left out of subsequent subgroup analysis to avoid confounding interpretation of results.

\section{Enrichment Analysis}

The hypergeometric sampling distribution provides an estimate for the probability of observing a given number of overlapping elements, based on the number of elements in each set and the total number of elements each set was drawn from. This probability distribution was used to assess the statistical significance of overlaps observed between the pairs of gene sets identified as distinguishing the 'malignant-' and 'benign-like' subgroups of borderline ovarian tumors from each of our data set and the Tothill et al data set, using the R function 'phyper'.

For Gene Ontology (GO) enrichment analysis, lists of Affymetrix probe-set IDs were uploaded to the DAVID functional analysis tool. ${ }^{21}$ Significance of enrichment was inferred from Benjamini-Hochberg adjusted $P$-values from the GO Biological Process ontology.

For exploring cell adhesion-related functional signatures in the 'borderline-classification' gene sets, the 'GO.db' and 'hgu133plus2' packages from Bioconductor were used to obtain annotation information. Enrichments from KEGG pathways (hsa04514, hsa04510, hsa04512 and hsa04530) and GO terms (GO: 0007155; GO: 0016477; GO: 005923; GO: 0005925) were evaluated using the hypergeometric test.

\section{Results}

\section{Expression Profiling Reveals Subtypes of Borderline Ovarian Tumors}

To investigate structure within the data set relating to similarities between serous benign, borderline and low-grade malignant tumors samples, unsupervised hierarchical clustering was performed on the data set. Clustering (Figure 1a) shows that the samples form two main groups: one group (lefthand branch) includes all malignant samples and the other group (right-hand branch) includes all benign samples, whereas borderline ovarian tumors are distributed between these two distinct groups.

Unsupervised hierarchical clustering was performed for all genes on borderline ovarian tumors only. Clustering (Figure 1b) shows two main groups of borderline ovarian tumors, with only three samples that do not clearly fit into either group. The main group in the left-hand branch of Figure 1b 
a

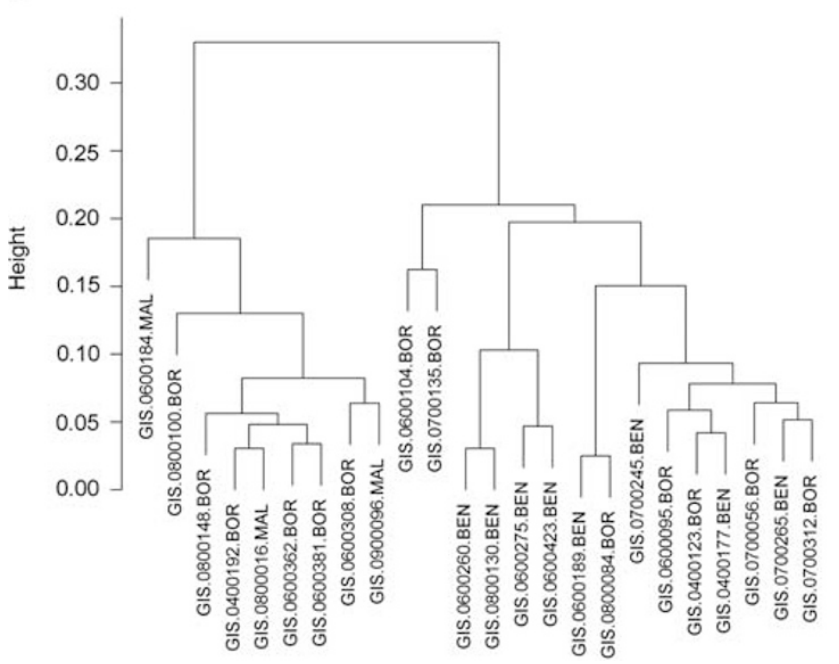

b

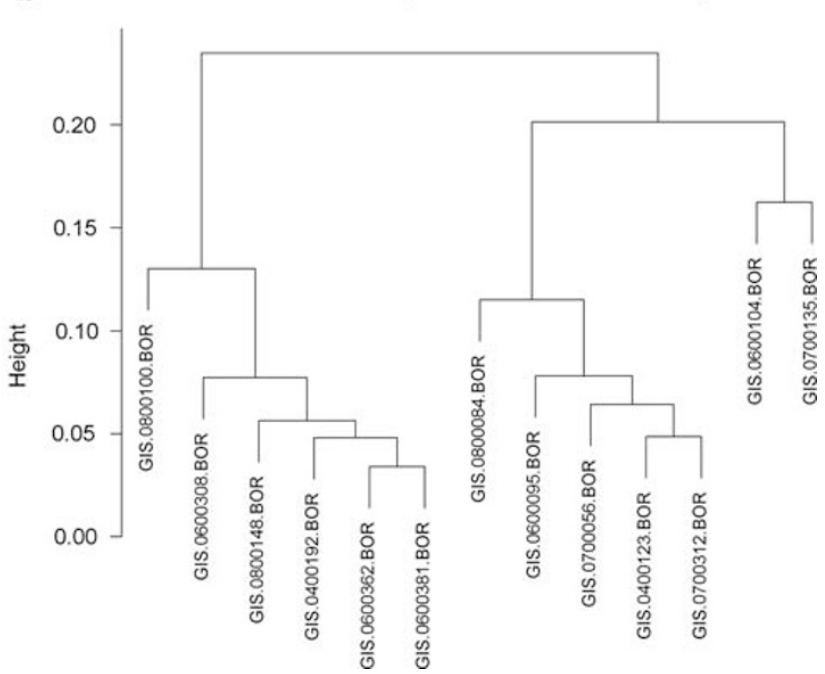

Figure 1 Dendrograms produced from unsupervised hierarchical clustering of all serous ovarian lesions (a) and borderline tumors only (b) from our data set, based on correlation of genome-wide expression profiles. For each sample, a (non-identifiable) patient number and a malignancy indicator are shown. 'BEN' indicates that the sample came from a benign lesion, 'BOR' indicates that the sample came from a borderline tumor and 'MAL' indicates that the sample came from a low-grade serous epithelial ovarian carcinoma.

contains only borderline ovarian tumors that clustered with benign lesions in Figure 1a, whereas the group clustering on the right-hand side of Figure $1 \mathrm{~b}$ borderline ovarian tumors that clustered with malignant tumors.

To validate these observations, we interrogated a published data set from an independent cohort, the Tothil et al data set, which included 18 serous borderline ovarian tumors and 3 grade 1 serous carcinomas. Unsupervised hierarchical clustering of these samples was performed using the normalized gene expression data (Figure 2). Although this cohort contained no benign lesions, it is clear from Figure 2 that the borderline ovarian tumors (labeled 'LMP' in this plot) form two distinct groups, and one of those groups (the right-hand branch) is devoid of malignant samples, whereas the other group (the large central branch) includes low-grade malignant samples. This mirrors the dichotomization of borderline ovarian tumors seen in our data set, in which there was a 'malignant-like' subgroup of borderline ovarian tumors, but it remains conjecture at this point that the subgroup of borderline ovarian tumors in the Tothill et al data set that clustered distinctly from the malignant samples represent a more 'benign-like' subgroup as was found in our data set.

\section{Expression of Malignancy-Associated Genes in Borderline Ovarian Tumors Subgroups}

We hypothesized that if the subgroups of borderline ovarian tumors we identified were associated with different levels of molecular similarity to benign or malignant tumors, repeating the hierarchical clus-

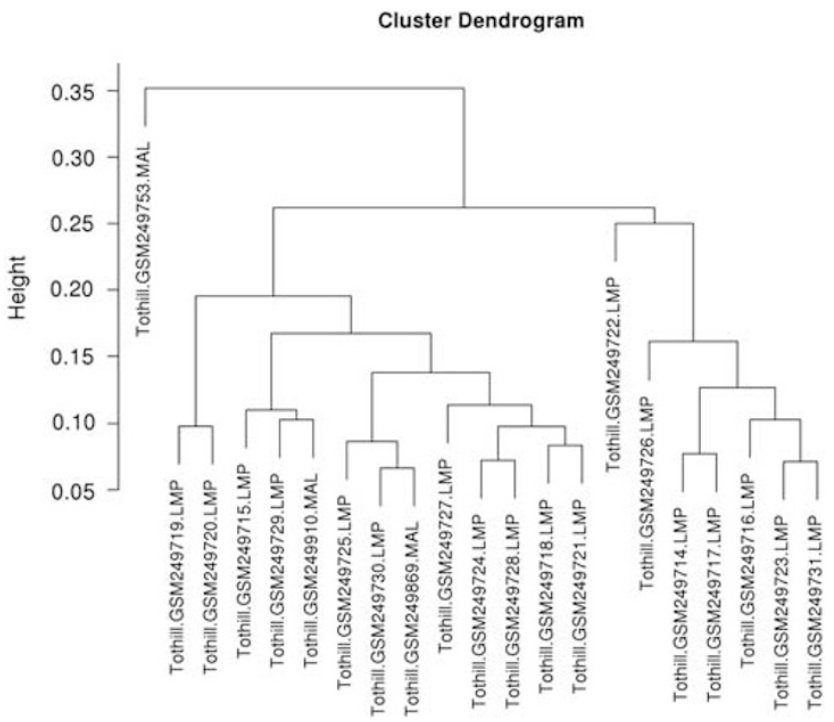

Figure 2 Unsupervised hierarchical clustering of serous ovarian tumors from the Tothill et al data set, based on correlation of genome-wide expression profiles. For each sample, a (nonidentifiable) patient number and a malignancy indicator are shown. 'LMP' indicates that the sample came from a borderline tumor and 'MAL' indicates that the sample came from a low-grade serous epithelial ovarian carcinoma.

tering of borderline ovarian tumors using only genes that were differentially expressed between the benign and low-grade serous malignant tumors would exaggerate the dichotomization.

A list of 1602 probe sets with significant differential expression between the benign and low-grade malignant samples of the data set was obtained (this gene 


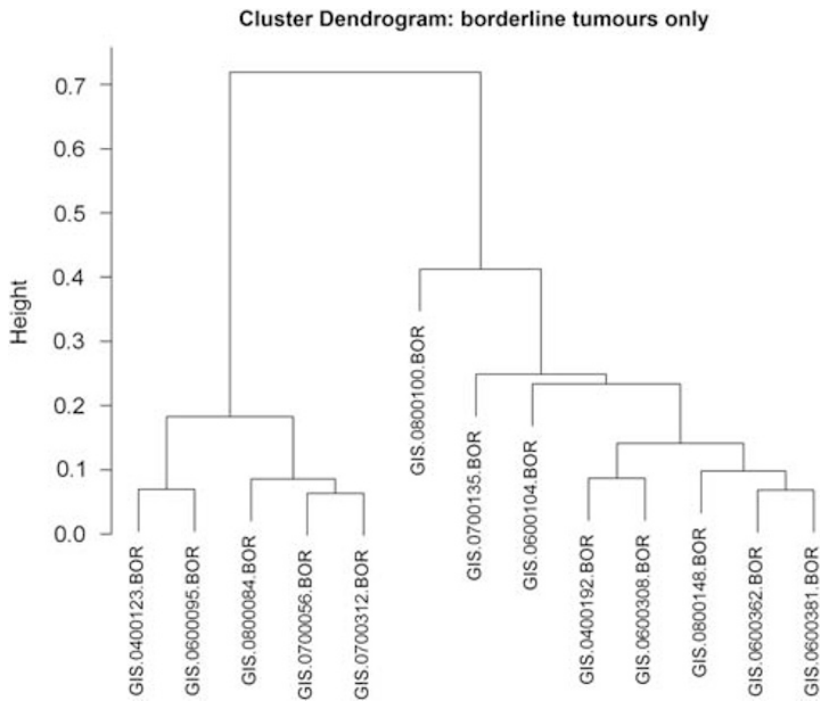

Figure 3 Hierarchical clustering of borderline tumors based on correlation of expression profiles across genes that were significantly differentially expressed between benign and low-grade malignant serous tumors. The (non-identifiable) patient number is shown for each sample.

set is given in Supplementary Table S2). Figure 3 shows the result of hierarchical clustering of borderline ovarian tumors in terms of only these genes. Interestingly, the groupings shown in Figure 3 are nearly identical to those shown in Figure 1b, but the distances between the samples within each group are greater in Figure 3. This suggests that the malignancyassociated genes capture the main distinction between the observed subgroups of similar borderline ovarian tumors, further supporting the case that these subgroups reflect 'benign-' and 'malignant-like' borderline ovarian tumors.

\section{Characterization of Molecular Distinctions Between Subgroups of Borderline Tumors}

To find genes that characterized the benign- and malignant-like molecular distinctions between the subgroups of borderline ovarian tumors, it was necessary to define reliable sets of benign- and malignant-like subgroups. Consensus subgroups were defined resulting in a set of five benign-like and a set of five malignant-like borderline ovarian tumors.

With consensus malignancy-associated subgroups of borderline ovarian tumors, LIMMA was used to identify genes with significant differential expression between the two subgroups. There were 410 probe sets (subsequently referred to as the 'benignlike gene set') with significantly higher expression in the benign-like subgroup and 312 probe sets (subsequently referred to as the 'malignant-like gene set') with significantly higher expression in the malignant-like subgroup. These gene sets are given in Supplementary Table S3. A heatmap showing expression of the gene sets across borderline ovarian tumors is provided in Figure 4.
If these genes characterize a general biological distinction between subgroups of borderline ovarian tumors, it could reasonably be expected that the subgroups of borderline ovarian tumors from the Tothill et al data set would display patterns of expression similar to the corresponding subgroup from our data set. A heatmap, showing expression levels across the two borderline ovarian tumors subgroups of the Tothill et al data set of the borderline-classification gene sets identified from our data set is given in Figure 5. The clustering of the samples in Figure 5 preserves the dichotomization of the complete transcriptional profiles of the samples (Figure 2), demonstrating that the principal transcriptional features distinguishing the two subgroups of borderline ovarian tumors in our data set capture the main transcriptional distinctions between the subgroups of borderline ovarian tumors in an independent validation cohort.

For a statistical assessment of this similarity, the genes best distinguishing the two subgroups of borderline ovarian tumors in the Tothill et al data set were identified, following the same procedure as in the identification of our borderline ovarian tumor classification gene sets. The significance of the overlap between the 'malignant-like gene set' identified from each data set was evaluated using the hypergeometric test, giving $P<1 \times 10^{-65}$. Evaluating the overlap between the 'benign-like gene set' identified from each data set gave $P<1 \times 10^{-5}$.

CA125 is widely used as a serum-based clinical marker for ovarian cancer. ${ }^{22}$ It is therefore interesting to note that the gene encoding CA125 (MUC16) was expressed at significantly higher levels in the malignant-like subgroup of borderline ovarian tumors in the Tothill et al data set than the corresponding benign-like subgroup of borderline ovarian tumors ( $t$-test $P=0.001$ ). The equivalent comparison between the subgroups of borderline ovarian tumors from our data set revealed a similar trend of higher CA125 expression in the malignantlike subgroup when compared with the benign-like subgroup ( $t$-test $P=0.06$ ).

\section{Towards Gene Expression-Based Prediction of Malignant Potential of Borderline Ovarian Tumors}

The 'combined-classifier' gene sets that are significantly differentially expressed (in the same direction) between the two subgroups of borderline ovarian tumors are given in Supplementary Table S4, comprising 8 probe sets (mapping to 5 unique genes) with higher expression in 'benign-like' borderline ovarian tumors and 50 probe sets (mapping to 45 unique genes) with higher expression in 'malignant-like' borderline ovarian tumors. Heatmaps of the expression of the genes belonging to the 'combined-classifier' gene set across the classified borderline ovarian tumors of each data set are given in Figure 6, showing the clear dichotomization of 

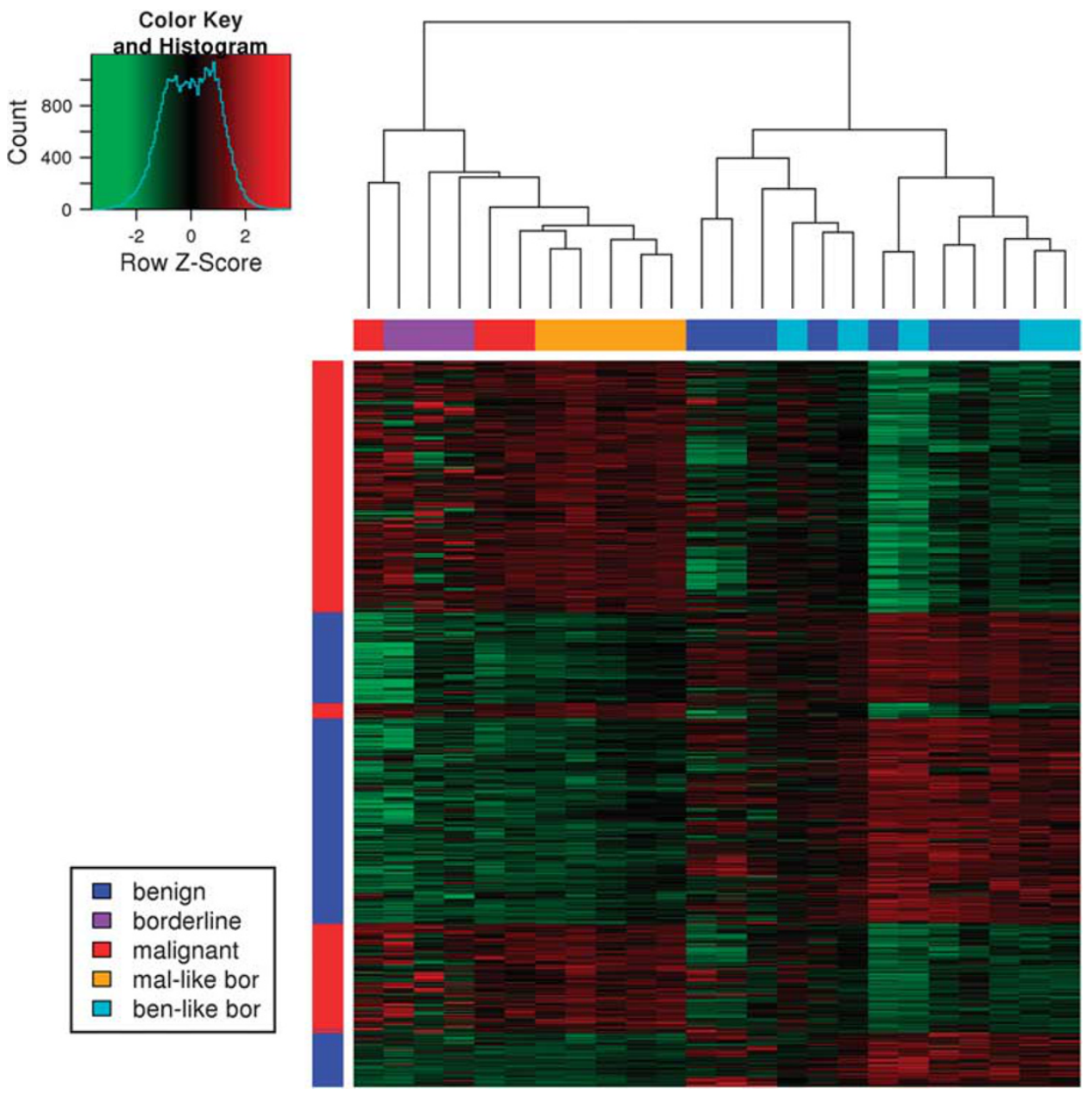

Figure 4 Heatmap showing relative expression levels of borderline-classification genes, across all samples in the data set. Red indicates higher expression level and green indicates lower expression. Row-side color panel indicates which genes are malignant-like borderline associated (orange) or benign-like borderline associated (cyan). Color panel along the top of heatmap indicates which samples are lowgrade serous carcinomas (red), benign lesions (blue), benign-like borderline tumors (cyan) and malignant-like borderline tumors (orange). Samples labeled purple are borderline tumors not reliably clustering into either of the two subgroups.

the different subgroups of borderline ovarian tumors in each data set. Inspection of the classification according to each probe set individually reveals that the majority of the individual probe sets, comprising this gene set, separate the borderline ovarian tumors of each data set perfectly into their subgroups.

\section{Different Modes of Cell Adhesion Characterize Borderline Ovarian Tumors Subgroups}

Using DAVID to perform functional enrichment analysis of the borderline-classification gene sets derived from our study, it was found that GO terms associated with cell adhesion were highly enriched in borderline ovarian tumors than the malignantand the benign-like gene set (Supplementary Table S5). Furthermore, the GO terms 'Cell Motion' (GO: 0006928) and 'Cell Motility' (GO: 0016477) were enriched in the malignant-like gene set with Benjamini-Hochberg adjusted $P$-values 0.002 and
0.007, respectively, but not enriched in the benignlike gene set. We therefore sought to characterize the differences in transcriptional control of cell adhesion between the benign- and malignant-like borderline ovarian tumors.

A set of individual GO and KEGG pathway terms was used to investigate enrichments in the borderline-classifier gene sets from borderline ovarian tumors studies, as described in Materials and methods. The KEGG pathway 'Cell Adhesion' (hsa04514) was significantly enriched in the 'malignant-like' gene set from the combined classifier $(P=0.04)$, with $C L D N 10$ contributing to the signature. Claudin proteins are essential for tight junction formation, and the GO 'tight junction' term (GO: 0005923) is significantly enriched in borderline ovarian tumor 'malignant-like' gene sets $(P=0.05$ for the gene set derived from the Tothill data set and $P=0.02$ for the gene set derived from our data set), but not the respective benign-like gene sets. This 

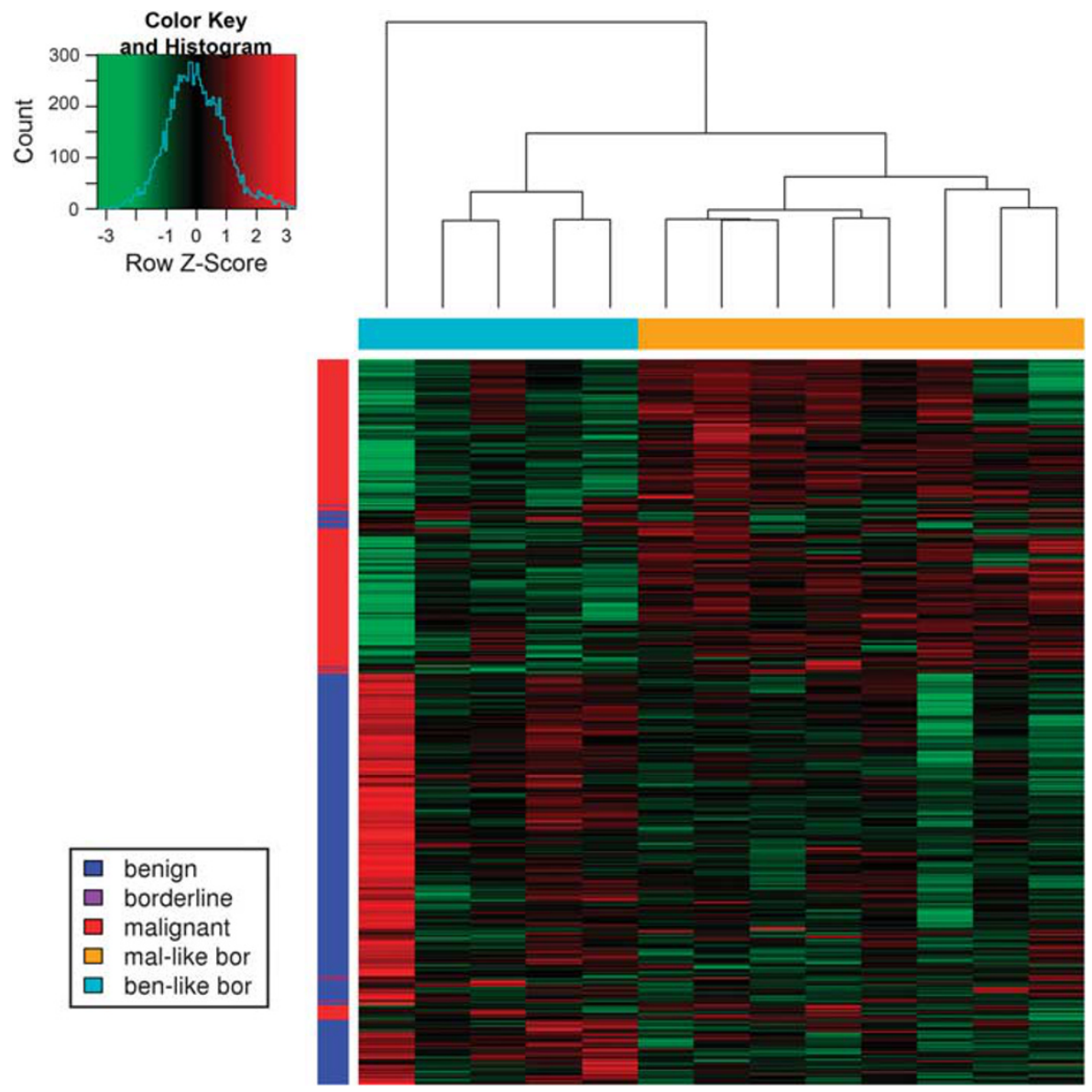

Figure 5 Heatmap showing relative expression levels of borderline-classification genes, across serous borderline ovarian tumor of the Tothill et al data set. Red indicates higher expression level and green indicates lower expression. Row-side color panel indicates which genes are malignant-like borderline associated (red) or benign-like borderline associated (blue). Color panel along the top of heatmap indicates which samples are the hypothesized benign-like borderline tumors (cyan) and the malignant-like borderline tumors (orange), as identified from unsupervised hierarchical clustering. Borderline tumor samples not clearly classified as either benign-like or malignantlike were excluded.

bias towards Claudin-based tight junctions in the malignant-like borderline ovarian tumors, when compared with benign-like borderline ovarian tumors, is further supported by the fact that $C L D N 3$ contributed to the 'malignant-like' cell adhesion signature in our data set, and in borderline ovarian tumors CLDN4 and CLDN7 were expressed at significantly higher levels in the malignant-like borderline ovarian tumors subgroup in the Tothill et al data set than the corresponding benign-like borderline ovarian tumors subgroup.

\section{Relationship Between Molecular Signature and Histopathological Features}

In our set of borderline ovarian tumors no relationship was found between a benign- and malignantlike tumor signature and tumor stage or the presence of non-invasive implants. However, the two tumors showing microinvasion and micropapillary pattern (0800100 and 600381) consistently appeared closer to malignant tumors.

\section{Discussion}

Borderline ovarian tumors are a challenging group of ovarian tumors positioned between benign and malignant neoplasms. We have recently reported the results of profiling the DNA methylomes of serous benign, borderline and low-grade serous carcinoma. Consensus clustering highlighted differences between borderline ovarian tumors methylomes and returned subgroups with malignant- or benign-like methylation profiles. ${ }^{23}$

In this study, we compared the gene expression profile of a different cohort of benign, borderline and low-grade malignant serous tumors. Unsupervised hierarchical clustering revealed distinct segregation between benign tumors and low-grade serous carcinoma. Borderline ovarian tumors were separated between the two groups, with a few cases clustering independently. Repeating the clustering of borderline ovarian tumors using only genes differentially expressed between benign tumors and low-grade serous carcinoma exaggerated the 

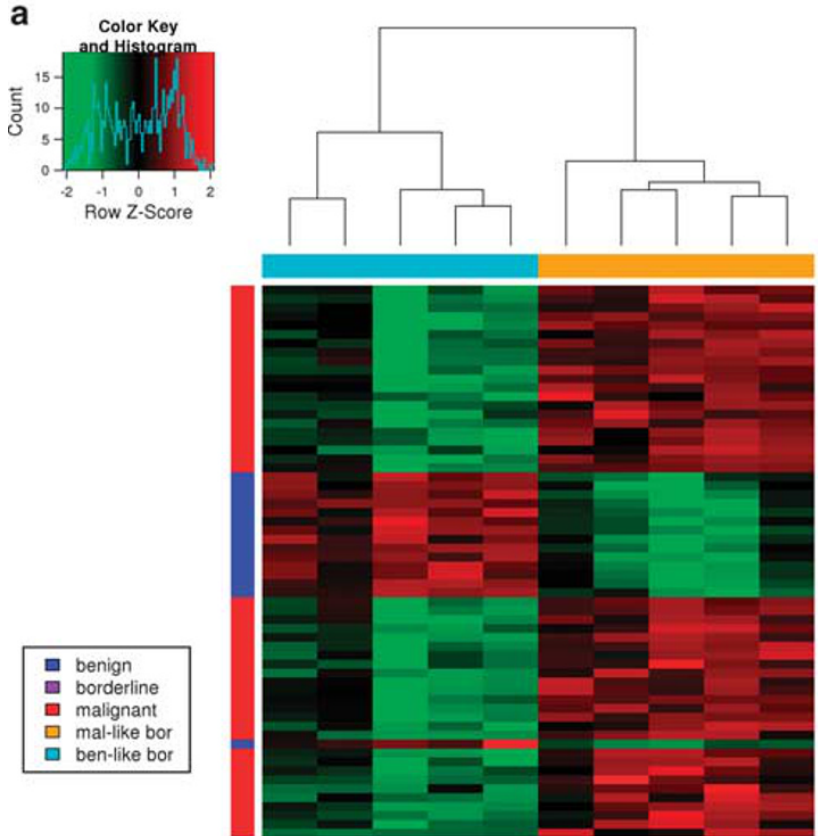

b
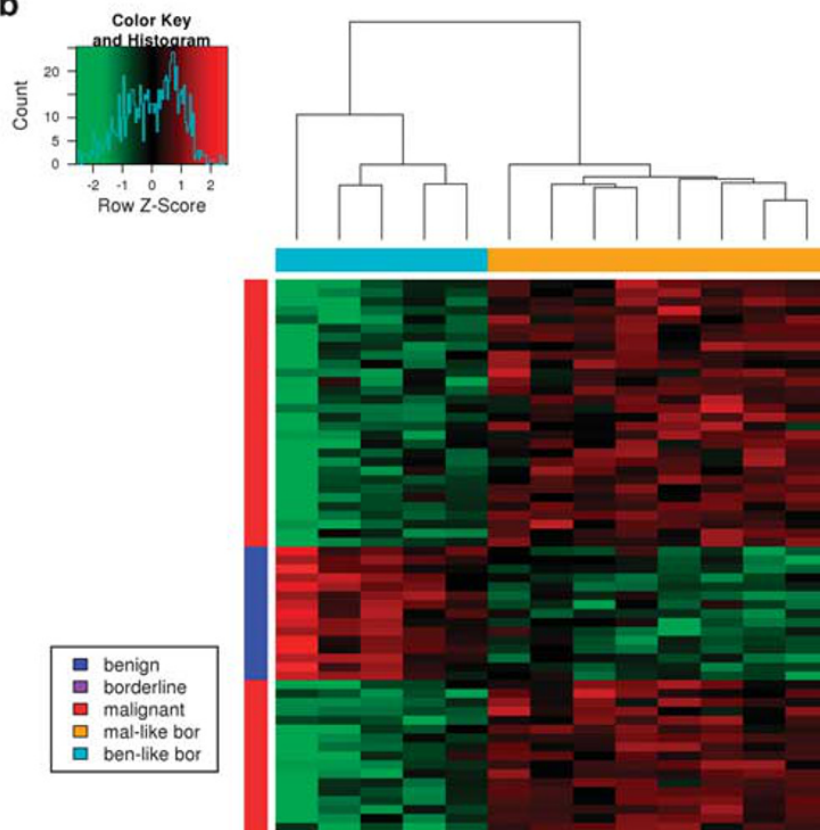

Figure 6 Heatmaps showing relative expression levels of the 'combined-classifier' genes, across serous borderline ovarian tumor of our data set (a) and the Tothill et al data set (b). Red indicates higher expression level and green indicates lower expression. Row-side color panel indicates which genes are malignant-like borderline associated (red) or benign-like borderline associated (blue). Color panel along the top of heatmap indicates which samples are the hypothesized benign-like borderline tumors (cyan) and the malignant-like borderline tumors (orange), as identified from unsupervised hierarchical clustering. For each data set, borderline tumor samples not clearly classified as either benign-like or malignant-like were excluded.

dichotomization, confirming that this separation is quite robust. This additionally suggests that the malignancy-associated genes capture the main distinction between the observed subgroups of borderline ovarian tumors.

To validate our finding, we interrogated a published data set from an independent cohort. ${ }^{19}$ The borderline ovarian tumors in the Tothill et al data set cluster into two distinct groups, with one group devoid of malignant tumors and the other including low-grade serous carcinoma. In the Tothill et al data set, the 'malignant-like' borderline ovarian tumors express genes from our 'malignant-like gene set' at a higher level than the other subgroup of borderline ovarian tumors, and this other subgroup of borderline ovarian tumors express genes from our 'benign-like gene set' at a higher level than the malignant-like borderline ovarian tumors. This confirms that the subgroup of borderline ovarian tumors from the Tothill et al data set that are not associated with low-grade serous carcinoma are likely to represent a 'benign-like' subgroup. This demonstrates that transcriptional features distinguishing the two subgroups of borderline ovarian tumors in our data set capture the main transcriptional distinctions between the subgroups of borderline ovarian tumors in an independent validation cohort.

Interestingly, in borderline ovarian tumors data sets the 'malignant-like' subgroup of borderline ovarian tumors expressed CA125 at significantly higher levels than the 'benign-like' borderline ovarian tumors, showing that the identified subgroups of borderline ovarian tumors show different levels of expression for this marker, which is commonly used as a serum marker for ovarian cancer. ${ }^{22}$

Functional enrichment analysis shows that genes encoding components of tight junctions were significantly enriched in the sets of genes that were expressed at higher levels in the malignant-like borderline ovarian tumors subgroups of borderline ovarian tumors data sets, when compared with their respective benign-like borderline ovarian tumors subgroup. This is a relevant finding as components of the tight junction, which were significantly overexpressed in the malignant-like borderline ovarian tumors subgroups, namely the Claudins CLDN3, CLDN4 and CLDN7, have been shown to promote migration and invasion of ovarian carcinoma cells. Borderline ovarian tumors and low-grade serous carcinoma do not respond well to conventional chemotherapy. Suppression of CLDN4 has been shown to result in a significant increase of cisplatin sensitivity. CLDN4 expression was significantly greater in ovarian cancer tissue from chemoresistant patients compared with chemosensitive patients. These data suggest that CLDN4 contributes to platinum resistance in ovarian cancer and may be a potential target in the treatment of platinum-resistant tumors. ${ }^{24}$ 
Bonome et $a l^{16}$ have shown that based on gene expression profiling borderline ovarian tumors are distinct from high-grade serous carcinoma, and remarkably similar to low-grade serous carcinoma. Our study shows that not all borderline ovarian tumors share this similarity and that some are distinct from low-grade serous carcinoma and show more similarity to benign tumors. A minority of borderline ovarian tumors failed to cluster with either benign or malignant tumors in borderline ovarian tumor studied data sets. Although this may be due to technical reasons, it might also suggest that there is a further, subgroup of borderline ovarian tumors at the molecular level.

In 1996 Seidman and Kurman ${ }^{25}$ proposed abolishing the category of borderline ovarian tumors and subclassifying these tumors into benign and malignant types, where the benign subgroup is composed of typical serous borderline ovarian tumors, including those with non-invasive implants, whereas tumors displaying a micropapillary growth pattern and tumors with invasive implants should be classified as carcinomas. ${ }^{25}$ Our data in principle support this proposal with some reservations. At the molecular level, there appears to be two subsets of borderline ovarian tumors, one benign-like and one malignant-like. Reviewing the histopathological features of tumors in borderline ovarian tumors subgroups did not reveal significant distinctive features between the two groups that allow segregation on morphological grounds mirroring the molecular profiling. However, the case showing micropapillary architecture clustered with the malignant-like group, which is in support of Seidman's proposal. However, cases associated with non-invasive implants appeared to be equally distributed between subgroups, indicating that cases with no implants or with non-invasive implants cannot be entirely dismissed as benign-like on histological basis alone.

Tumor stage is believed to be the most important prognostic marker in patients with borderline ovarian tumors, ${ }^{26}$ yet it was not one of the parameters proposed by Seidman and Kurman to classify borderline ovarian tumors as benign and malignant on histological basis. Our data show that tumor stage does not seem to show correlation with molecular subtype in borderline ovarian tumors.

Microinvasion was not used by Seidman and Kurman ${ }^{26}$ as one of the discriminating parameters of benign and malignant borderline ovarian tumors. In our data set, the two cases with microinvasion clustered with the malignant-like subgroup, suggesting that microinvasion is a step on the transformation to frankly invasive disease borderline ovarian tumors at the histological and molecular level. In support of that is the fact that GO annotations of the differentially expressed genes between the two molecular subgroups of borderline ovarian tumors point to cell motility and cell adhesion signatures, borderline ovarian tumors of which have pivotal roles in tumor invasion and metastasis, hallmarks of malignancy. The findings suggest that microinvasion in borderline ovarian tumors may not be as innocuous as formerly thought, and that it might be a histologic feature that could assist in the subclassification of these tumors.

We identified 50 genes that accurately classified borderline ovarian tumors into subgroups in the two independent data sets. This set of genes could provide the basis for identifying biomarkers to predict the malignant potential of borderline ovarian tumors, which would have considerable clinical impact in the management of these tumors.

This study confirms the presence of molecular subtypes of borderline ovarian tumors, substantiating our recent results from DNA methylation profiling. ${ }^{23}$ Our findings present a new concept and provide the basis for further research and validation of the clinical relevance of this molecular classification. More cases need to be studied, with correlation of the molecular classification with clinical data to validate the molecular classification as a means of clinically stratifying serous borderline ovarian tumors. Clarification of the molecular changes in borderline ovarian tumors and validation of their clinical correlations is critical for developing optimal, evidence-based clinical management algorithms for these tumors.

\section{Acknowledgements}

We acknowledge funding from Ovarian Cancer Action, as well as support from Imperial Experimental Cancer Research and Biomedical Research Centers. We thank Prof. Edison T Liu, Genome Institute of Singapore and the Agency for Science Technology and Research of Singapore for supporting the generation of microarray expression data.

\section{Disclosure/conflict of interest}

The authors have no conflict of interest.

\section{References}

1 Lenhard MS, Mitterer S, Kumper C, et al. Long-term follow-up after ovarian borderline tumor: relapse and survival in a large patient cohort. Eur J Obstet Gynecol Reprod Biol 2009;145:189-194.

2 Jones MB. Borderline ovarian tumors: current concepts for prognostic factors and clinical management. Clin Obstet Gynecol 2006;49:517-525.

3 Manek S, Wells M. Pathology of borderline ovarian tumours. Clin Oncol (R Coll Radiol) 1999;11:73-77.

4 Morice P, Camatte S, Rey A, et al. Prognostic factors for patients with advanced stage serous borderline tumours of the ovary. Ann Oncol 2003;14:592-598.

5 Seidman JD, Kurman RJ. Ovarian serous borderline tumors: a critical review of the literature with 
emphasis on prognostic indicators. Hum Pathol 2000; 31:539-557.

6 Longacre TA, McKenney JK, Tazelaar HD, et al. Ovarian serous tumors of low malignant potential (borderline tumors): outcome-based study of 276 patients with long-term ( $>$ or $=5$-year) follow-up. Am J Surg Pathol 2005;29:707-723.

7 McKenney JK, Balzer BL, Longacre TA. Patterns of stromal invasion in ovarian serous tumors of low malignant potential (borderline tumors): a reevaluation of the concept of stromal microinvasion. Am J Surg Pathol 2006;30:1209-1221.

8 Shih Ie M, Kurman RJ. Ovarian tumorigenesis: a proposed model based on morphological and molecular genetic analysis. Am J Pathol 2004;164:1511-1518.

9 Kurman RJ, Shih Ie M. The origin and pathogenesis of epithelial ovarian cancer: a proposed unifying theory. Am J Surg Pathol 2010;34:433-443.

10 Giordano G, Azzoni C, D'Adda T, et al. Human papilloma virus (HPV) status, p16INK4a, and p53 overexpression in epithelial malignant and borderline ovarian neoplasms. Pathol Res Pract 2008;204: 163-174.

11 Dehari R, Kurman RJ, Logani S, et al. The development of high-grade serous carcinoma from atypical proliferative (borderline) serous tumors and low-grade micropapillary serous carcinoma: a morphologic and molecular genetic analysis. Am J Surg Pathol 2007;31:1007-1012.

12 Singer G, Oldt R 3rd, Cohen Y, et al. Mutations in BRAF and KRAS characterize the development of lowgrade ovarian serous carcinoma. J Natl Cancer Inst 2003;95:484-486.

13 Zanotti KM, Hart WR, Kennedy AW, et al. Allelic imbalance on chromosome 17p13 in borderline (low malignant potential) epithelial ovarian tumors. Int J Gynecol Pathol 1999;18:247-253.

14 Micci F, Haugom L, Ahlquist $\mathrm{T}$, et al. Genomic aberrations in borderline ovarian tumors. J Transl Med 2010;8:21.

15 Ortiz BH, Ailawadi M, Colitti C, et al. Second primary or recurrence? Comparative patterns of p53 and K-ras mutations suggest that serous borderline ovarian tumors and subsequent serous carcinomas are unrelated tumors. Cancer Res 2001;61:7264-7267.

16 Bonome T, Lee JY, Park DC, et al. Expression profiling of serous low malignant potential, low-grade, and high-grade tumors of the ovary. Cancer Res 2005; 65:10602-10612.

17 May T, Virtanen C, Sharma M, et al. Low malignant potential tumors with micropapillary features are molecularly similar to low-grade serous carcinoma of the ovary. Gynecol Oncol 2010;117:9-17.

18 Irizarry RA, Hobbs B, Collin F, et al. Exploration, normalization, and summaries of high density oligonucleotide array probe level data. Biostatistics 2003; 4:249-264.

19 Tothill RW, Tinker AV, George J, et al. Novel molecular subtypes of serous and endometrioid ovarian cancer linked to clinical outcome. Clin Cancer Res 2008; 14:5198-5208.

20 Smyth GK. Linear models and empirical bayes methods for assessing differential expression in microarray experiments. Stat Appl Genet Mol Biol 2004;3:ISSN (Online) 1544-6115.

21 Huang da W, Sherman BT, Lempicki RA. Systematic and integrative analysis of large gene lists using DAVID bioinformatics resources. Nat Protoc 2009;4:44-57.

22 Olt G, Berchuck A, Bast RC Jr. The role of tumor markers in gynecologic oncology. Obstet Gynecol Surv 1990;45:570-577.

23 Zeller C, Dai W, Curry E, et al. The DNA methylomes of serous borderline tumors reveal subgroups with malignant- or benign-like profiles. Am J Pathol 2013;182: 668-677.

24 Yoshida H, Sumi T, Zhi X, et al. Claudin-4: a potential therapeutic target in chemotherapy-resistant ovarian cancer. Anticancer Res 2011;31:1271-1277.

25 Seidman JD, Kurman RJ. Subclassification of serous borderline tumors of the ovary into benign and malignant types. A clinicopathologic study of 65 advanced stage cases. Am J Surg Pathol 1996;20: 1331-1345.

26 Hogg R, Scurry J, Kim SN, et al. Microinvasion links ovarian serous borderline tumor and grade 1 invasive carcinoma. Gynecol Oncol 2007;106:44-51.

Supplementary Information accompanies the paper on Modern Pathology website (http://www.nature.com/ modpathol) 